\title{
The $\gamma^{*} \gamma^{*}$ total cross section in next-to-leading order BFKL and LEP2 data
}

\author{
D.Yu. Ivanov, ${ }^{a}$ B. Murdaca ${ }^{b}$ and A. Papa ${ }^{b}$ \\ a Sobolev Institute of Mathematics and Novosibirsk State University, \\ 630090 Novosibirsk, Russia \\ ${ }^{b}$ Dipartimento di Fisica, Università della Calabria, and Istituto Nazionale di Fisica Nucleare, \\ Gruppo collegato di Cosenza, Arcavacata di Rende, I-87036 Cosenza, Italy \\ E-mail: d-ivanov@math.nsc.ru, beatrice.murdaca@fis.unical.it, \\ alessandro.papa@fis.unical.it
}

Abstract: We study the total cross section for the collision of two highly-virtual photons at large energies, taking into account the BFKL resummation of energy logarithms with full next-to-leading accuracy. A necessary ingredient of the calculation, the next-to-leading order impact factor for the photon to photon transition, has been calculated by Balitsky and Chirilli using an approach based on the operator expansion in Wilson lines. We extracted the result for the photon impact factor in the original BFKL calculation scheme comparing the expression for the photon-photon total cross section obtained in BFKL with the one recently derived by Chirilli and Kovchegov in the Wilson-line operator expansion scheme.

We perform a detailed numerical analysis, combining different, but equivalent in nextto-leading accuracy, representations of the cross section with various optimization methods of the perturbative series. We compare our results with previous determinations in the literature and with the LEP2 experimental data. We find that the account of Balitsky and Chirilli expression for the photon impact factor reduces the BFKL contribution to the cross section to very small values, making it impossible to describe LEP2 data as the sum of BFKL and leading-order QED quark box contributions.

KeYwORDS: Resummation, QCD

ArXiv EPRINT: 1407.8447 


\section{Contents}

1 Introduction 1

2 BFKL contribution to the $\gamma^{*} \gamma^{*}$ total cross section 3

3 Numerical analysis $\quad 6$

$\begin{array}{lll}3.1 & \text { Chirilli-Kovchegov representation } & 7\end{array}$

3.2 Series representation with PMS optimization 9

3.3 Exponential representation with PMS optimization 9

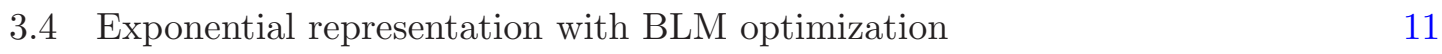

4 Discussion $\quad 13$

\section{Introduction}

Similarly to the $e^{+} e^{-}$annihilation into hadrons, the total cross section for the collision of two off-shell photons with large virtualities is an important test ground for perturbative QCD. At a fixed order of $\alpha_{s}$ and at low energies, the dominant contribution comes from the quark box, calculated at the leading-order (LO) in refs. [1, 2] (see figure 1) and at the next-to-LO (NLO) in ref. [3]. In ref. $[4,5]$ the resummation of double logs appearing in the NLO corrections to the quark box was also studied. At higher energies, the gluon exchange in the $t$-channel overwhelms the quark exchange contribution, due to the different power asymptotics for $s \rightarrow \infty$. At higher orders in $\alpha_{s}$, the contributions from $t$-channel gluons lead to terms with powers of single logarithms of the energy, which must be resummed.

The BFKL approach [6-9] provides for a consistent theoretical framework for the resummation of the energy logarithms, both in the leading logarithmic approximation (LLA), which means resummation of all terms $\left(\alpha_{s} \ln (s)\right)^{n}$, and in the next-to-leading approximation (NLA), which means resummation of all terms $\alpha_{s}\left(\alpha_{s} \ln (s)\right)^{n}$. In this approach, the imaginary part of the amplitude (and, hence, the total cross section) for a large- $s$ hard collision process can be written as the convolution of the Green's function of two interacting Reggeized gluons with the impact factors of the colliding particles (see figure 2).

The study of the $\gamma^{*} \gamma^{*}$ total cross section in LLA BFKL has a long history [10-20]. For the extension of these results to the NLA level one needs to consider corrections to both the BFKL Green's function and to the impact factors of colliding virtual photons.

The Green's function is determined through the BFKL equation and is process-independent. The NLO kernel of the BFKL equation for singlet color representation in the $t$-channel and forward scattering, relevant for the determination of a total cross section in the NLA, has been achieved in refs. [21, 22], after the long program of calculation of the NLO corrections [23-36] (for a review, see ref. [37]). 

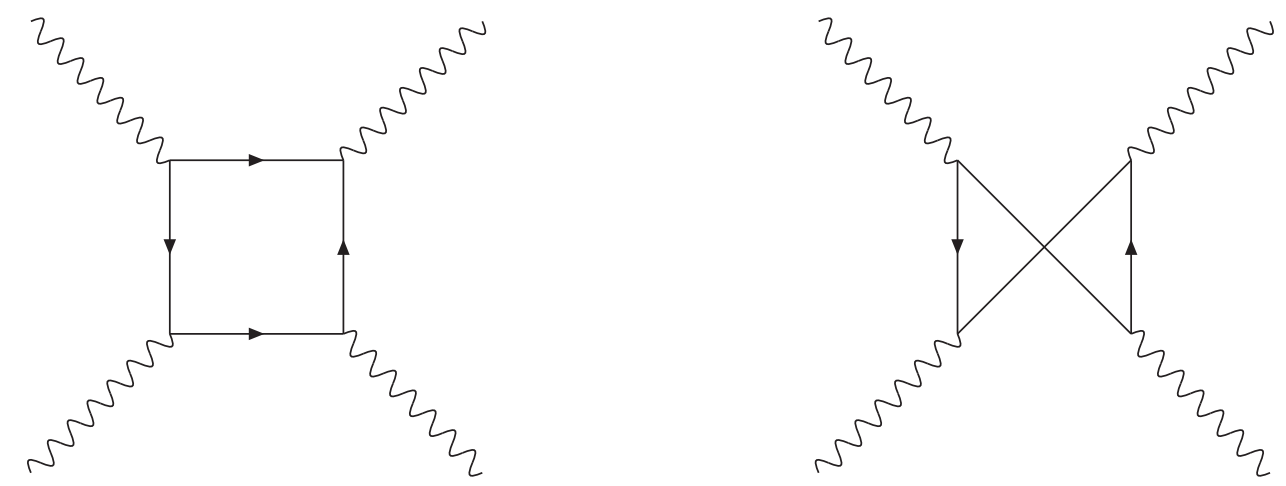

Figure 1. Quark box LO diagrams.

The other essential ingredient for the $\gamma^{*} \gamma^{*}$ total cross section is the impact factor for the virtual photon to virtual photon transition. While its LO expression is known since long, the NLO calculation, carried out in the momentum representation, turned out to be rather complicated and was completed only after year-long efforts [38-44]. The lengthy result was published over a few years in pieces, some of them available only in the form of a numerical code, thus making it of limited practical use. Indeed, until very recently, the inclusion of BFKL resummation effects in the NLA calculation of the $\gamma^{*} \gamma^{*}$ total cross section was carried out only in approximate way, by taking the BFKL Green's function in the NLA while using the LO expression for impact factors. This is the case of the pioneer paper in ref. [45] (see also ref. [46]) and of the later analysis in refs. [47] and [48].

The situation changed radically recently, when the NLO photon impact was calculated in the coordinate space and then transformed to the momentum representation and to the Mellin (or $\gamma$-representation) [49] (see also ref. [50]). The NLO expression for the photon impact factor turns out to be very simple in all representations, thus confirming an already well established evidence (see, for instance, refs. [51-53]) that the use of the coordinate representation leads to much simpler expressions for the NLO BFKL kernel and impact factors, which, in the momentum representation, would be the result of not so obvious cancellations.

Now all ingredients are available to build the $\gamma^{*} \gamma^{*}$ total cross with full inclusion of the BFKL resummation in the NLA. Indeed, already in ref. [50] there is a first numerical estimate of the $\gamma^{*} \gamma^{*}$ total cross section in the NLA. Note that, the derivation of the results for the $\gamma^{*} \gamma^{*}$ total cross section in ref. [50] follows closely the approach developed earlier in ref. [54], where the high-energy limit of $\mathcal{N}=4$ SYM amplitudes was considered. Besides, the authors of ref. [50] used their formulas for the eigenfunctions of the NLO BFKL kernel derived in ref. [55].

As a matter of fact, previous studies of physical processes within the BFKL approach in the NLA, such as the photoproduction of two light vector mesons [56-59] and the production of Mueller-Navelet jets [60-67], have clearly shown that NLA expressions for an observable (such as a cross-section or an azimuthal correlation), though being formally equivalent up to subleading terms, may lead to somewhat different numerical estimates. 


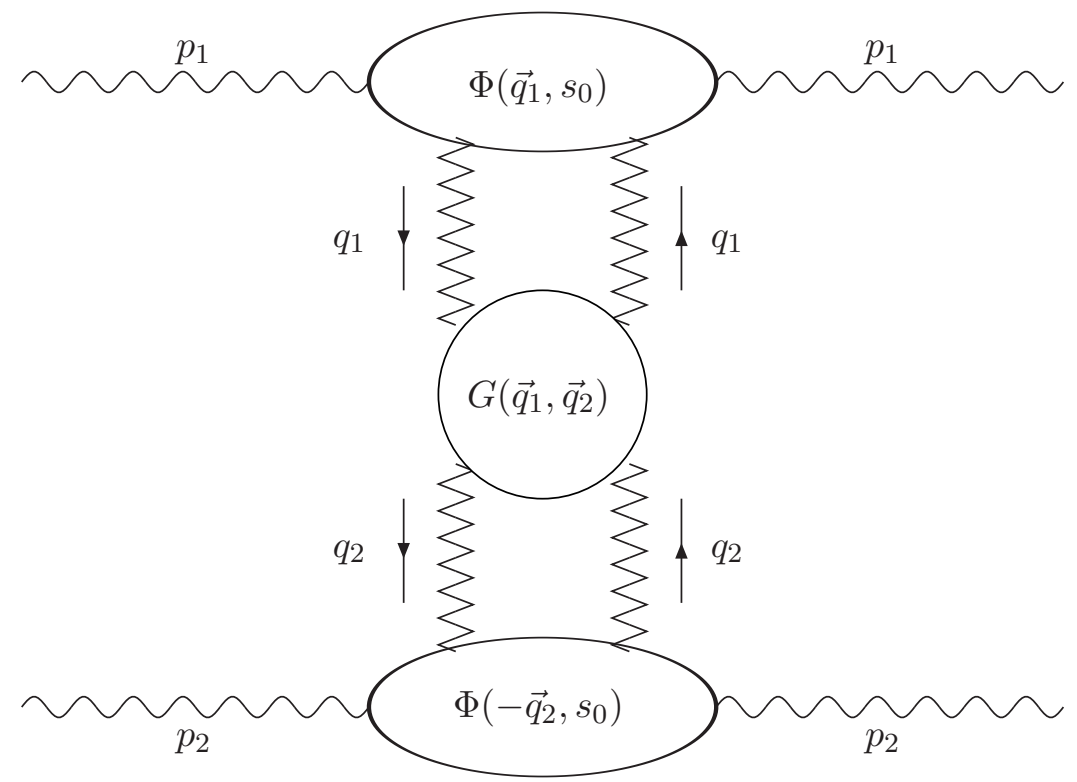

Figure 2. Schematic representation of the elastic amplitude for the $\gamma^{*}\left(p_{1}\right) \gamma^{*}\left(p_{2}\right)$ forward scattering.

At the basis of this observation is the fact that NLO BFKL corrections, both of the kernel and of impact factors, are typically of opposite sign with respect to the LO and large in absolute value. For this reason a numerical estimate cannot be reliable (i) if some optimization procedure for the perturbative series is not applied and (ii) if not corroborated by a careful numerical analysis, aimed at assessing the stability of the result under variation of the original NLA expressions for the observable of interest within a large enough class of NLA-equivalent expressions.

The aim of this paper is to contribute to such analysis, by comparing several NLAequivalent representations of the $\gamma^{*} \gamma^{*}$ total cross section, in combination with two among the most common methods of optimization of the perturbative series, namely the principle of minimal sensitivity (PMS) $[68,69]$ and the Brodsky-Lepage-Mackenzie (BLM) method [70]. Moreover, the results of this analysis will be contrasted with the only experimental data available so far, obtained at LEP2 [71, 72].

The paper is organized as follows: in section 2 we present the general structure of the $\gamma^{*} \gamma^{*}$ total cross section in the NLA and, by comparison with refs. [49, 50], extract the NLO photon impact factor in the original BFKL calculation scheme; in section 3 we use this information to build several NLA-equivalent representations of the cross section and present, for each of them, the behavior with the energy in comparison with the LEP2 experimental data; finally, in section 4, we discuss our results and draw the conclusions.

\section{BFKL contribution to the $\gamma^{*} \gamma^{*}$ total cross section}

The total cross section of two unpolarized photons with virtualities $Q_{1}$ and $Q_{2}$ can be obtained from the imaginary part of the forward amplitude. In LLA BFKL and in the Mellin-representation (also said $\gamma$ - or $\nu$-representation), it is given by the following expres- 
sion (see, for instance, ref. [45]):

$$
\sigma_{\text {tot }}^{\gamma^{*} \gamma^{*}}\left(s, Q_{1}, Q_{2}\right)=\sum_{i, k=T, L} \frac{1}{(2 \pi)^{2} Q_{1} Q_{2}} \int_{-\infty}^{+\infty} d \nu\left(\frac{Q_{1}^{2}}{Q_{2}^{2}}\right)^{i \nu} F_{i}(\nu) F_{k}(-\nu)\left(\frac{s}{s_{0}}\right)^{\bar{\alpha}_{s} \chi(\nu)}
$$

where $\bar{\alpha}_{s} \equiv \alpha_{s}\left(\mu_{R}\right) N_{c} / \pi$, with $N_{c}$ the number of colors, $\chi(\nu)$ is the so-called characteristic BFKL function,

$$
\chi(\nu)=2 \psi(1)-\psi\left(\frac{1}{2}+i \nu\right)-\psi\left(\frac{1}{2}-i \nu\right)
$$

and

$$
\begin{aligned}
F_{T}(\nu)=F_{T}(-\nu) & =\alpha \alpha_{s}\left(\sum_{q} e_{q}^{2}\right) \frac{\pi}{2} \frac{\left(\frac{3}{2}-i \nu\right)\left(\frac{3}{2}+i \nu\right) \Gamma^{2}\left(\frac{1}{2}-i \nu\right) \Gamma^{2}\left(\frac{1}{2}+i \nu\right)}{\Gamma(2-i \nu) \Gamma(2+i \nu)} \\
& =\alpha \alpha_{s}\left(\sum_{q} e_{q}^{2}\right) \frac{\pi^{2}}{8} \frac{9+4 \nu^{2}}{\nu\left(1+\nu^{2}\right)} \frac{\sinh ^{2}(\pi \nu)}{\cosh ^{2}(\pi \nu)}, \\
F_{L}(\nu)=F_{L}(-\nu) & =\alpha \alpha_{s}\left(\sum_{q} e_{q}^{2}\right) \pi \frac{\Gamma\left(\frac{3}{2}-i \nu\right) \Gamma\left(\frac{3}{2}+i \nu\right) \Gamma\left(\frac{1}{2}-i \nu\right) \Gamma\left(\frac{1}{2}+i \nu\right)}{\Gamma(2-i \nu) \Gamma(2+i \nu)} \\
& =\alpha \alpha_{s}\left(\sum_{q} e_{q}^{2}\right) \frac{\pi^{2}}{4} \frac{1+4 \nu^{2}}{\nu\left(1+\nu^{2}\right)} \frac{\sinh (\pi \nu)}{\cosh ^{2}(\pi \nu)}
\end{aligned}
$$

are the LO impact factors for transverse and longitudinal polarizations, respectively. In the previous equations, $\alpha$ is the electromagnetic coupling constant, the summation extends over all active quarks (taken massless) and $e_{q}$ is the quark electric charge in units of the electron charge. In the expression (2.1) for the LLA BFKL cross section the argument of the strong and electromagnetic coupling constants, $\mu_{R}$, and the value of the scale $s_{0}$ are not fixed.

Following the procedure of refs. $[57,58]$, it is possible to write down the NLA BFKL cross section as follows:

$$
\begin{aligned}
& \sigma_{\text {tot }}^{\gamma^{*} \gamma^{*}}\left(s, Q_{1}, Q_{2}, s_{0}, \mu_{R}\right)=\frac{1}{(2 \pi)^{2} Q_{1} Q_{2}} \int_{-\infty}^{+\infty} d \nu\left(\frac{Q_{1}^{2}}{Q_{2}^{2}}\right)^{i \nu}\left(\frac{s}{s_{0}}\right)^{\bar{\alpha}_{s}\left(\mu_{R}\right) \chi(\nu)} \\
& \times \sum_{i, k=T, L} F_{i}(\nu) F_{k}(-\nu)\left\{1+\bar{\alpha}_{s}\left(\mu_{R}\right)\left(\frac{F_{i}^{(1)}\left(\nu, s_{0}, \mu_{R}\right)}{F_{i}(\nu)}+\frac{F_{k}^{(1)}\left(-\nu, s_{0}, \mu_{R}\right)}{F_{k}(-\nu)}\right)\right. \\
& \left.\quad+\bar{\alpha}_{s}^{2}\left(\mu_{R}\right) \ln \left(\frac{s}{s_{0}}\right)\left[\bar{\chi}(\nu)+\frac{\beta_{0}}{8 N_{c}} \chi(\nu)\left(-\chi(\nu)+\frac{10}{3}+2 \ln \frac{\mu_{R}^{2}}{Q_{1} Q_{2}}\right)\right]\right\},
\end{aligned}
$$

where

$$
\begin{aligned}
\bar{\chi}(\nu)= & -\frac{1}{4}\left[\frac{\pi^{2}-4}{3} \chi(\nu)-6 \zeta(3)-\chi^{\prime \prime}(\nu)-\frac{\pi^{3}}{\cosh (\pi \nu)}\right. \\
& \left.+\frac{\pi^{2} \sinh (\pi \nu)}{2 \nu \cosh ^{2}(\pi \nu)}\left(3+\left(1+\frac{n_{f}}{N_{c}^{3}}\right) \frac{11+12 \nu^{2}}{16\left(1+\nu^{2}\right)}\right)+4 \phi(\nu)\right],
\end{aligned}
$$




$$
\phi(\nu)=2 \int_{0}^{1} d x \frac{\cos (\nu \ln (x))}{(1+x) \sqrt{x}}\left[\frac{\pi^{2}}{6}-\operatorname{Li}_{2}(x)\right], \quad \operatorname{Li}_{2}(x)=-\int_{0}^{x} d t \frac{\ln (1-t)}{t},
$$

$n_{f}$ is the number of active quarks, $F_{L, T}^{(1)}\left(\nu, s_{0}, \mu_{R}\right)$ are the NLO corrections to the longitudinal/transverse photon impact factor in the $\nu$-representation and

$$
\beta_{0}=\frac{11}{3} N_{c}-\frac{2}{3} n_{f} .
$$

Note that our notations are slightly different in comparison to the ones used in refs. [57, 58]. The impact factors which we introduced here differ by some factors from the impact factors $c_{1,2}$ (and $\left.c_{1,2}^{(1)}\right)$ of refs. [57, 58]:

$$
\begin{aligned}
c_{1, i}(\nu) & =\left(Q_{1}^{2}\right)^{i \nu-1 / 2} F_{i}(\nu) \\
c_{2, k}(\nu) & =\left(Q_{2}^{2}\right)^{-i \nu-1 / 2} F_{k}(-\nu) .
\end{aligned}
$$

Moreover, in the derivation of the last term of eq. (2.5) the symmetry property of the LO photon impact factors, $F_{L, T}(\nu)=F_{L, T}(-\nu)$, was used.

Our goal now is to extract the NLO parts of the photon impact factors, $F_{L, T}^{(1)}\left(\nu, s_{0}, \mu_{R}\right)$, which enter the cross section eq. (2.5) in the original BFKL approach, by comparing eq. (2.5) with the results for the $\gamma^{*} \gamma^{*}$ cross section obtained recently in the Wilson-line operator expansion scheme by Chirilli and Kovchegov [50]. According to eqs. (3.40) and (3.41) of ref. [50], the cross section in the case of transverse and longitudinal polarizations reads

$$
\begin{aligned}
\sigma_{T T}^{(\mathrm{CK})}= & \left(\sum_{q} e_{q}^{2}\right)^{2} \frac{\alpha^{2} \alpha_{s}^{2}}{Q_{1} Q_{2}} \frac{\pi^{2}}{2^{8}} \int_{-\infty}^{+\infty} d \nu\left(\frac{Q_{1}^{2}}{Q_{2}^{2}}\right)^{i \nu}\left(\frac{s}{Q_{1} Q_{2}}\right)^{\bar{\alpha}_{s} \chi(\nu)+\bar{\alpha}_{s}^{2} \chi^{(1)}(\nu)} \\
& \times\left[\frac{\left(9+4 \nu^{2}\right)}{\nu\left(1+\nu^{2}\right)} \frac{\sinh (\pi \nu)}{\cosh ^{2}(\pi \nu)}\right]^{2}\left[1+\frac{\alpha_{s}}{\pi}+\frac{\bar{\alpha}_{s}}{2} \mathcal{F}_{1}(\nu)\right]\left[1+\frac{\alpha_{s}}{\pi}+\frac{\bar{\alpha}_{s}}{2} \mathcal{F}_{1}(-\nu)\right] \\
& \times\left\{1+\bar{\alpha}_{s} \Re[F(\nu)]\right\} \\
\sigma_{L L}^{(\mathrm{CK})}= & \left(\sum_{q} e_{q}^{2}\right)^{2} \frac{\alpha^{2} \alpha_{s}^{2}}{Q_{1} Q_{2}} \frac{\pi^{2}}{2^{8}} \int_{-\infty}^{+\infty} d \nu\left(\frac{Q_{1}^{2}}{Q_{2}^{2}}\right)^{i \nu}\left(\frac{s}{Q_{1} Q_{2}}\right)^{\bar{\alpha}_{s} \chi(\nu)+\bar{\alpha}_{s}^{2} \chi^{(1)}(\nu)} \\
& \times\left[\frac{\left(9+4 \nu^{2}\right)}{\nu\left(1+\nu^{2}\right)} \frac{\sinh ^{2}(\pi \nu)}{\cosh ^{2}(\pi \nu)}\right]^{2}\left[\frac{11+12 \nu^{2}}{9+4 \nu^{2}}\left(1+\frac{\alpha_{s}}{\pi}+\frac{\bar{\alpha}_{s}}{2} \mathcal{F}_{2}(\nu)\right)\right. \\
& \left.-\left(1+\frac{\alpha_{s}}{\pi}+\frac{\bar{\alpha}_{s}}{2} \mathcal{F}_{1}(\nu)\right)\right]\left[\frac{11+12 \nu^{2}}{9+4 \nu^{2}}\left(1+\frac{\alpha_{s}}{\pi}+\frac{\bar{\alpha}_{s}}{2} \mathcal{F}_{2}(-\nu)\right)\right. \\
& \left.-\left(1+\frac{\alpha_{s}}{\pi}+\frac{\bar{\alpha}_{s}}{2} \mathcal{F}_{1}(-\nu)\right)\right]\left\{1+\bar{\alpha}_{s} \Re[F(\nu)]\right\}
\end{aligned}
$$

where in the r.h.s. the strong coupling without argument stands for the coupling at the symmetric point, $\alpha_{s}=\alpha_{s}\left(\sqrt{Q_{1} Q_{2}}\right), \Re[F(\nu)]$ is given in eq. (3.37) of ref. [50] and the explicit expressions for $\mathcal{F}_{1,2}(\nu)$ in eq. (52) of ref. [49]; for the definition of $\chi^{(1)}(\nu)$, see eqs. (2.9) and (2.11) of ref. [50], so that

$$
\chi^{(1)}(\nu)=\bar{\chi}(\nu)+\frac{\beta_{0}}{8 N_{c}} \chi(\nu)\left(-\chi(\nu)+\frac{10}{3}\right) .
$$


Note that working with NLA accuracy, we replaced in eqs. (2.9) and (2.10) the original factors $\alpha_{s}\left(Q_{1}\right) \alpha_{s}\left(Q_{2}\right)$ present in eqs. (3.40) and (3.41) of ref. [50] by $\alpha_{s}^{2}\left(\sqrt{Q_{1} Q_{2}}\right)$, since

$$
\alpha_{s}\left(Q_{1}\right) \alpha_{s}\left(Q_{2}\right)=\alpha_{s}^{2}\left(\sqrt{Q_{1} Q_{2}}\right)+\mathcal{O}\left(\alpha_{s}^{4}\right) .
$$

Another point is that the terms $\alpha_{s} / \pi$ which appear in the r.h.s. of eqs. (2.9) and (2.10) are due to the QCD vacuum polarization contribution, which actually reads as $3 C_{F} \alpha_{s} /(4 \pi)$, where $C_{F}=\left(N_{c}^{2}-1\right) /\left(2 N_{c}\right){ }^{1}$

Now we are ready to compare eqs. (2.9) and (2.10) with the BFKL cross section eq. (2.5) taken for the particular choice of scales $\mu_{R}^{2}=s_{0}=Q_{1} Q_{2}$. Expanding eqs. (2.9) and (2.10) into a form similar to eq. (2.5) and requiring the coincidence of the two representations for the cross section with NLA accuracy allows us to extract without ambiguity the NLO parts of the BFKL impact factors $F_{L, T}^{(1)}\left(\nu, s_{0}, \mu_{R}\right)$ (at the scale setting $s_{0}=\mu_{R}^{2}=Q_{1} Q_{2}$ ):

$$
\begin{aligned}
\frac{F_{T}^{(1)}\left(\nu, s_{0}, \mu_{R}\right)}{F_{T}(\nu)}= & \frac{\chi(\nu)}{2} \ln \frac{s_{0}}{Q^{2}}+\frac{\beta_{0}}{4 N_{c}} \ln \frac{\mu_{R}^{2}}{Q^{2}} \\
& +\frac{3 C_{F}}{4 N_{c}}-\frac{5}{18} \frac{n_{f}}{N_{c}}+\frac{\pi^{2}}{4}+\frac{85}{36}-\frac{\pi^{2}}{\cosh ^{2}(\pi \nu)}-\frac{4}{1+4 \nu^{2}}+\frac{6 \chi(\nu)}{9+4 \nu^{2}} \\
& +\frac{1}{2(1-2 i \nu)}-\frac{1}{2(1+2 i \nu)}-\frac{7}{18(3+2 i \nu)}+\frac{20}{3(3+2 i \nu)^{2}}-\frac{25}{18(3-2 i \nu)} \\
& +\frac{1}{2} \chi(\nu)\left[\psi\left(\frac{1}{2}-i \nu\right)+2 \psi\left(\frac{3}{2}-i \nu\right)-2 \psi(3-2 i \nu)-\psi\left(\frac{5}{2}+i \nu\right)\right]
\end{aligned}
$$

and

$$
\begin{aligned}
\frac{F_{L}^{(1)}\left(\nu, s_{0}, \mu_{R}\right)}{F_{L}(\nu)}= & \frac{\chi(\nu)}{2} \ln \frac{s_{0}}{Q^{2}}+\frac{\beta_{0}}{4 N_{c}} \ln \frac{\mu_{R}^{2}}{Q^{2}} \\
& +\frac{3 C_{F}}{4 N_{c}}-\frac{5}{18} \frac{n_{f}}{N_{c}}+\frac{\pi^{2}}{4}+\frac{85}{36}-\frac{\pi^{2}}{\cosh ^{2}(\pi \nu)}-\frac{8(1+4 i \nu)}{(1+2 i \nu)^{2}(1-2 i \nu)(3+2 i \nu)} \\
& +\frac{4}{3-4 i \nu+4 \nu^{2}} \chi(\nu) \\
& +\frac{1}{2} \chi(\nu)\left[\psi\left(\frac{1}{2}-i \nu\right)+2 \psi\left(\frac{3}{2}-i \nu\right)-2 \psi(3-2 i \nu)-\psi\left(\frac{5}{2}+i \nu\right)\right]
\end{aligned}
$$

The first lines of eqs. (2.12) and (2.13) describe the dependencies of the photon impact factors on the renormalization and energy scales, which are restored by the requirement that the BFKL cross section, eq. (2.5), does not depend on $s_{0}$ and $\mu_{R}$ with NLA accuracy.

\section{$3 \quad$ Numerical analysis}

In this section we are going to compare several different representations of the NLA $\gamma^{*} \gamma^{*}$ total cross section, which differ one from the other only by terms beyond the NLA. In

\footnotetext{
${ }^{1}$ We are very grateful to the authors of ref. [50] for the clarification of this issue and for establishing the overall normalization factor in their results for the cross section.
} 
a well-behaved perturbative series, the change of representation should not be numerically relevant. This is not the case in the BFKL framework, where it is well known that NLO corrections to kernel and impact factors are opposite in sign with respect to the LO contributions and large in absolute value.

It is very likely that also the (unknown) next-to-NLO corrections maybe opposite in sign with respect the NLO ones and large in absolute value, thus suggesting that fixing the BFKL energy scale $s_{0}$ and the renormalization scale $\mu_{R}$ at the "natural" values dictated by the kinematics of the process, i.e. $s_{0}=\mu_{R}^{2}=Q_{1} Q_{2}$, may well be not the best choice. For this reason, we will consider in the following two alternative procedures to fix the energy scales.

The first one is inspired by the PMS optimization method $[68,69]$ : for each value of the center-of-mass energy $s$ and of the virtualities of the colliding photons, we choose as optimal scales $s_{0}$ and $\mu_{R}$ those for which the given representation of the NLA cross section exhibits the minimum sensitivity under variation of these scales.

The other optimization procedure we consider is inspired by the BLM method [70]: again, for fixed $s$ and photon virtualities, we perform a finite renormalization to a momentum (MOM) scheme and then choose the renormalization scale $\mu_{R}$ in order to remove the $\beta_{0}$-dependent part in the given representation of the NLA cross section, while keeping the scale $s_{0}$ fixed at the natural value $Q_{1} Q_{2}$. In fact, there is some freedom in implementing the BLM optimization in this context and in the following we consider two different variants, dubbed $(a)$ and $(b)$, and give all necessary formulas, but relegate their derivation to a separate paper [73].

Below we will present predictions for the kinematic range relevant for the OPAL and L3 experiments at LEP2, considering equal photon virtualities, $Q_{1}=Q_{2} \equiv Q$, with $Q^{2}=17 \mathrm{GeV}^{2}$, and the energy range $Y=2 \div 6$, where $Y \equiv \ln \left(s / Q^{2}\right)$.

\subsection{Chirilli-Kovchegov representation}

As a first case, we try to apply to the description of LEP2 data the representation of the NLA $\gamma^{*} \gamma^{*}$ total cross section given in ref. [50]. It is given, with obvious meaning of the notation, by

$$
\sigma_{\text {tot }}^{(\mathrm{CK})}(s, Q)=\sigma_{T T}^{(\mathrm{CK})}+\sigma_{L L}^{(\mathrm{CK})}+\sigma_{T L}^{(\mathrm{CK})}+\sigma_{L T}^{(\mathrm{CK})}+\sigma_{\mathrm{LO} \text { box }}
$$

where we have included the LO contribution from the quark box, given in refs. [1, 2], and contributions of different polarization states of virtual photons. The explicit expressions for the first two terms in eq. (3.1) are given in eqs. (2.9) and (2.10) (for $Q_{1}=Q_{2}=Q$ ), the contributions of other polarizations can be obviously presented as follows:

$$
\begin{aligned}
\sigma_{T L}^{(\mathrm{CK})}= & \left(\sum_{q} e_{q}^{2}\right)^{2} \frac{\alpha^{2} \alpha_{s}^{2}}{Q^{2}} \frac{\pi^{2}}{2^{8}} \int_{-\infty}^{+\infty} d \nu\left(\frac{s}{Q^{2}}\right)^{\bar{\alpha}_{s} \chi(\nu)+\bar{\alpha}_{s}^{2} \chi^{(1)}(\nu)} \\
& \times\left[\frac{\left(9+4 \nu^{2}\right)}{\nu\left(1+\nu^{2}\right)} \frac{\sinh (\pi \nu)}{\cosh ^{2}(\pi \nu)}\right]^{2}\left[1+\frac{\alpha_{s}}{\pi}+\frac{\bar{\alpha}_{s}}{2} \mathcal{F}_{1}(\nu)\right]
\end{aligned}
$$




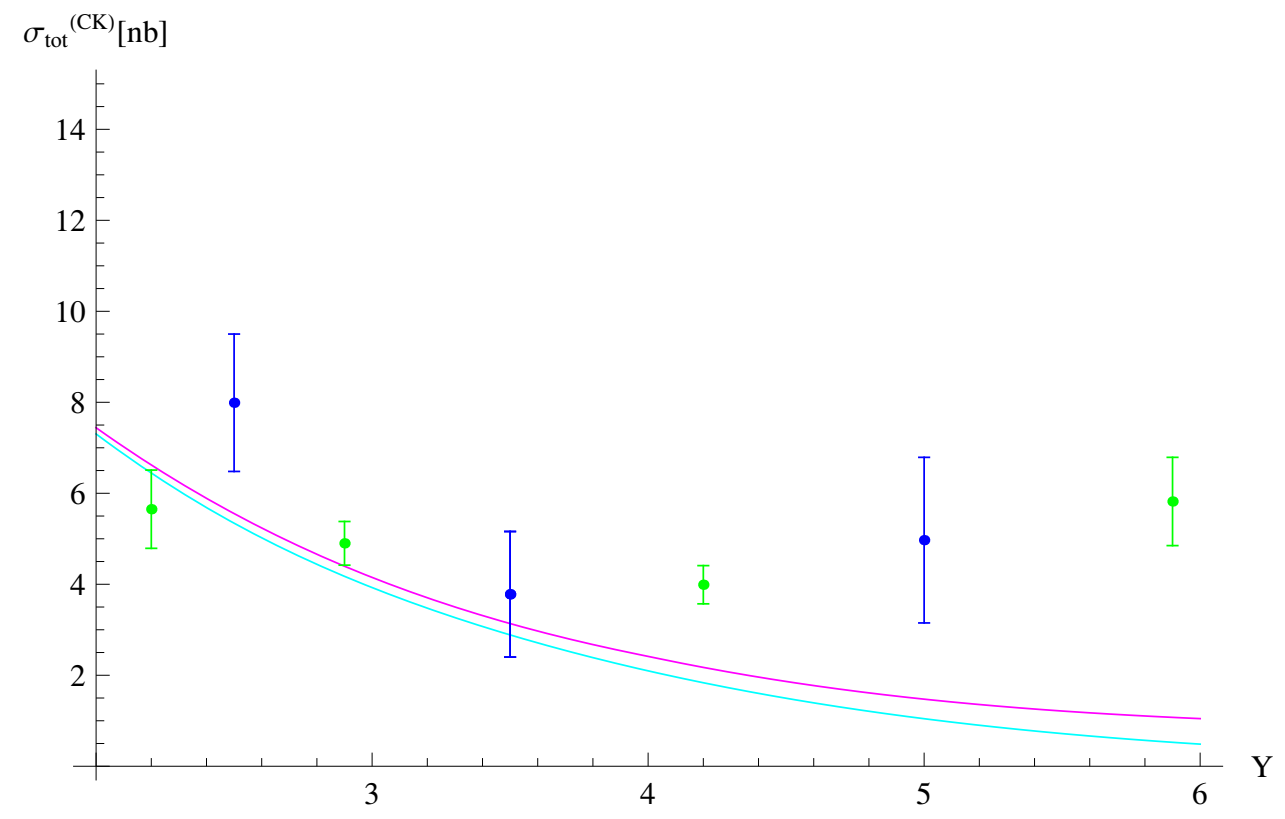

Figure 3. $\sigma_{\text {tot }}^{(\mathrm{CK})}$ versus $Y$ at $Q^{2}=17 \mathrm{GeV}^{2}\left(n_{f}=4\right)$ (magenta line), together with the experimental data from OPAL (blue points, $Q^{2}=18 \mathrm{GeV}^{2}$ ) and L3 (green points, $Q^{2}=16 \mathrm{GeV}^{2}$ ); the cyan line represents the LO quark box contribution only.

$$
\begin{aligned}
& \times\left[\frac{11+12 \nu^{2}}{9+4 \nu^{2}}\left(1+\frac{\alpha_{s}}{\pi}+\frac{\bar{\alpha}_{s}}{2} \mathcal{F}_{2}(-\nu)\right)\right. \\
& \left.-\left(1+\frac{\alpha_{s}}{\pi}+\frac{\bar{\alpha}_{s}}{2} \mathcal{F}_{1}(-\nu)\right)\right]\left\{1+\bar{\alpha}_{s} \Re[F(\nu)]\right\}, \\
\sigma_{L T}^{(\mathrm{CK})}= & \left(\sum_{q} e_{q}^{2}\right)^{2} \frac{\alpha^{2} \alpha_{s}^{2}}{Q^{2}} \frac{\pi^{2}}{2^{8}} \int_{-\infty}^{+\infty} d \nu\left(\frac{s}{Q^{2}}\right)^{\bar{\alpha}_{s} \chi(\nu)+\bar{\alpha}_{s}^{2} \chi^{(1)}(\nu)} \\
& \times\left[\frac{\left(9+4 \nu^{2}\right)}{\nu\left(1+\nu^{2}\right)} \frac{\sinh (\pi \nu)}{\cosh ^{2}(\pi \nu)}\right]^{2}\left[1+\frac{\alpha_{s}}{\pi}+\frac{\bar{\alpha}_{s}}{2} \mathcal{F}_{1}(-\nu)\right] \\
& \times\left[\frac{11+12 \nu^{2}}{9+4 \nu^{2}}\left(1+\frac{\alpha_{s}}{\pi}+\frac{\bar{\alpha}_{s}}{2} \mathcal{F}_{2}(\nu)\right)\right. \\
& \left.-\left(1+\frac{\alpha_{s}}{\pi}+\frac{\bar{\alpha}_{s}}{2} \mathcal{F}_{1}(\nu)\right)\right]\left\{1+\bar{\alpha}_{s} \Re[F(\nu)]\right\},
\end{aligned}
$$

where $\alpha_{s}=\alpha_{s}(Q), \mathcal{F}_{1,2}(\nu)$ and $\Re[F(\nu)]$ are given in eq. (52) of [49] and in eq. (3.37) of [50], respectively.

In figure 3 we report the behavior of $\sigma_{\text {tot }}^{(\mathrm{CK})}$ with $Y \equiv \ln \left(s / Q^{2}\right)$ for $Q^{2}=17 \mathrm{GeV}^{2}$ with $n_{f}=4$ and contrast it with the experimental data from CERN LEP2, namely three data points from OPAL [72] $\left(Q^{2}=18 \mathrm{GeV}^{2}\right)$ and four data points from L3 [71] $\left(Q^{2}=16 \mathrm{GeV}^{2}\right)$. We see that the original Chirilli-Kovchegov representation for the cross section (at natural values of the scales, $s_{0}=\mu_{R}^{2}=Q^{2}$ ) gives a very small BFKL contribution and does not agree well with data above $Y=4$.

In the following subsections, we are going to consider other representations of the cross section, equivalent to the Chirilli-Kovchegov one within the NLA, and admit the possibility 
of moving the energy scale $s_{0}$ and the renormalization scale $\mu_{F}$ from the "natural" kinematic value to some "optimal" scales, determined according the PMS or the BLM methods.

\subsection{Series representation with PMS optimization}

A convenient representation of the total cross section is the so-called "series representation", already used in refs. [57-59], which has the advantage of making manifest the BFKL resummation of leading and subleading energy logarithms and is very practical in numerical computations. It consists in writing the total cross section as follows

$$
\sigma_{\text {tot }}^{\text {(series) }}(s, Q)=\sigma_{T T}^{\text {(series) }}+\sigma_{L L}^{(\text {series })}+\sigma_{T L}^{(\text {series })}+\sigma_{L T}^{\text {(series) }}+\sigma_{\mathrm{LO} \text { box }},
$$

where for $i, k=L, T$

$$
Q^{2} \sigma_{i k}^{\text {(series) }}=\frac{1}{(2 \pi)^{2}}\left\{b_{0}^{i k}+\sum_{n=1}^{\infty} \bar{\alpha}_{s}^{n}\left(\mu_{R}\right) b_{n}^{i k}\left[\left(Y-Y_{0}\right)^{n}+d_{n}^{i k}\left(s_{0}, \mu_{R}\right)\left(Y-Y_{0}\right)^{n-1}\right]\right\},
$$

with $Y_{0} \equiv \ln \left(s_{0} / Q^{2}\right)$ and

$$
\begin{aligned}
b_{n}^{i k}= & \int_{-\infty}^{+\infty} d \nu F_{i}(\nu) F_{k}(-\nu) \frac{\chi^{n}(\nu)}{n !}, \\
d_{n}^{i k}= & n \ln \frac{s_{0}}{Q^{2}}+\frac{\beta_{0}}{4 N_{c}}\left[\frac{b_{n-1}^{i k}}{b_{n}^{i k}}\left((n+1) \ln \frac{\mu_{R}^{2}}{Q^{2}}+\frac{5}{3}(n-1)\right)-\frac{n(n-1)}{2}\right] \\
& +\frac{1}{b_{n}^{i k}} \int_{-\infty}^{+\infty} d \nu F_{i}(\nu) F_{k}(-\nu)\left[\frac{\chi^{n-1}(\nu)}{(n-1) !}\left(\frac{\bar{F}_{i}^{(1)}(\nu)}{F_{i}(\nu)}+\frac{\bar{F}_{k}^{(1)}(-\nu)}{F_{k}(-\nu)}\right)+\frac{\chi^{n-2}(\nu)}{(n-2) !} \bar{\chi}(\nu)\right],
\end{aligned}
$$

where we denoted for shortness $\bar{F}_{i}^{(1)}(\nu) \equiv F_{i}^{(1)}\left(\nu, s_{0}=Q^{2}, \mu_{R}=Q\right)$.

Our results for $\sigma_{\text {tot }}^{\text {(series) }}$ at $Q^{2}=17 \mathrm{GeV}^{2}$, obtained after truncation of the series at $n=40$, are summarized in table 1 , where we report, for each of the $Y$ values considered, also the optimal values of the energy scale $Y_{0}$ and renormalization scale $\mu_{R}$ found by the PMS method. In figure 4 we compare an interpolation of the data given in table 1 with the experimental data from LEP2 and with the result obtained in ref. [47] by the same method, but in the approximation where LO photon impact factors were used instead of NLO ones (i.e. the same approach as here, but with $\bar{F}_{i}^{(1)}(\nu) \rightarrow 0$ ). We observe that the large optimal values for the scales we find in this approach lead to a very low contribution to the cross section from the BFKL resummation and the overall scenario is basically the same as for the Chirilli-Kovchegov representation. We note that the big difference between this and the approximated result obtained in ref. [47] is a clear indication that the effect of NLO corrections to the impact factors is very substantial.

\subsection{Exponential representation with PMS optimization}

Here we consider representations of the NLA total cross section where the NLO corrections to the kernel are exponentiated, in two options, which differ by a subleading term given by the product of the two NLO corrections of the photon impact factors:

$$
\sigma_{\text {tot }}^{(\exp , 1)}(s, Q)=\sigma_{T T}^{(\exp , 1)}+\sigma_{L L}^{(\exp , 1)}+\sigma_{T L}^{(\exp , 1)}+\sigma_{L T}^{(\exp , 1)}+\sigma_{\mathrm{LO} \text { box }},
$$




\begin{tabular}{|llcc|}
\hline$Y$ & $\sigma_{\text {tot }}^{(\text {series })}[\mathrm{nb}]$ & $\mu_{R} / Q$ & $Y_{0}$ \\
\hline 2 & 7.3141 & 18 & 1 \\
3.5 & 3.1095 & 10 & 3 \\
4.5 & 1.9187 & 10 & 4 \\
6 & 1.1909 & 16 & 5 \\
\hline
\end{tabular}

Table 1. Values of $\sigma_{\text {tot }}^{\text {(series) }}$ for several values of $Y$ at $Q^{2}=17 \mathrm{GeV}^{2}$; the last two columns give the optimal values of the renormalization and energy scales.

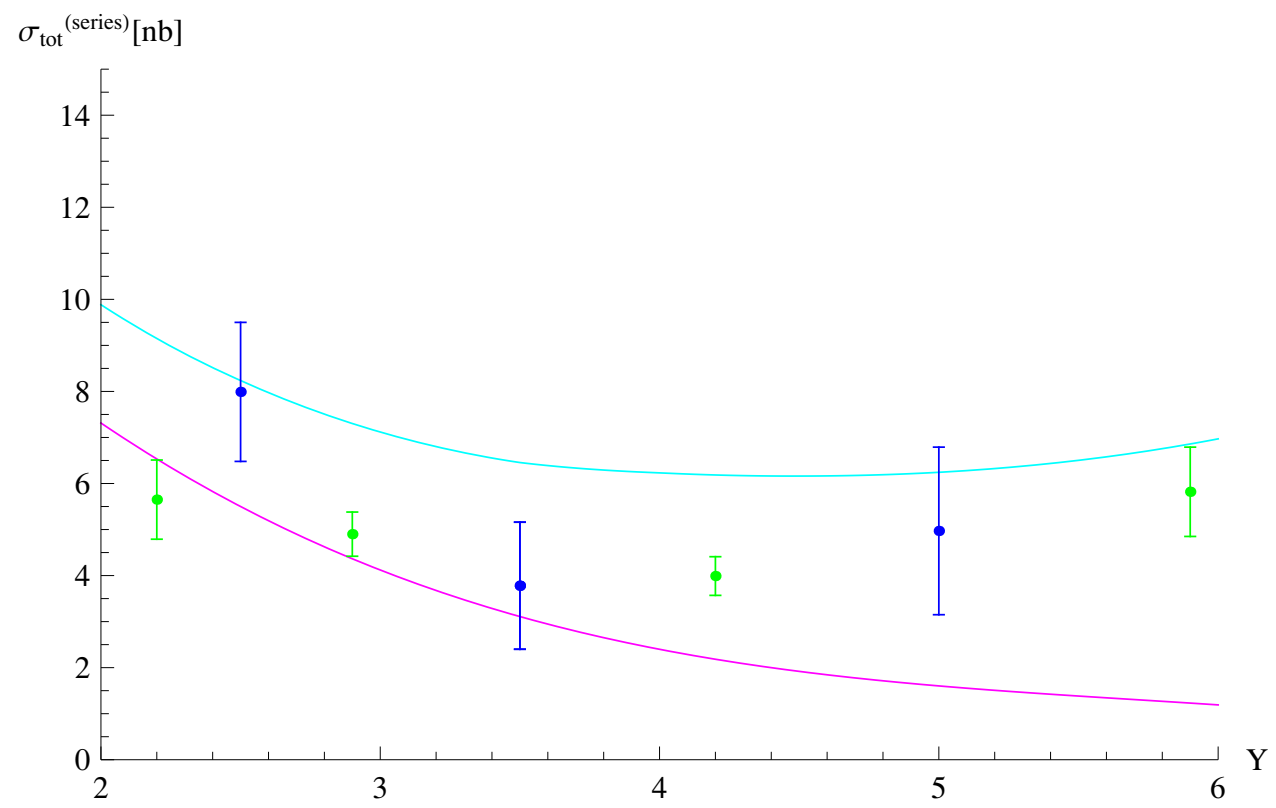

Figure 4. $\sigma_{\text {tot }}^{\text {(series) }}$ versus $Y$ at $Q^{2}=17 \mathrm{GeV}^{2}\left(n_{f}=4\right)$ (magenta line), together with the experimental data from OPAL (blue points, $Q^{2}=18 \mathrm{GeV}^{2}$ ) and L3 (green points, $Q^{2}=16 \mathrm{GeV}^{2}$ ); the cyan line represents the result of ref. [47] (see figure 3 there).

and

$$
\sigma_{\text {tot }}^{(\exp , 2)}(s, Q)=\sigma_{T T}^{(\exp , 2)}+\sigma_{L L}^{(\exp , 2)}+\sigma_{T L}^{(\exp , 2)}+\sigma_{L T}^{(\exp , 2)}+\sigma_{\mathrm{LO} \text { box }}
$$

with

$$
\begin{aligned}
\sigma_{i k}^{(\exp , 1)}= & \frac{1}{(2 \pi)^{2} Q^{2}} \int_{-\infty}^{+\infty} d \nu e^{\left(Y-Y_{0}\right)\left[\bar{\alpha}_{s}\left(\mu_{R}\right)\left(1+\frac{\bar{\alpha}_{s}\left(\mu_{R}\right) \beta_{0}}{4 N_{c}} \ln \frac{\mu_{R}^{2}}{Q^{2}}\right) \chi(\nu)+\bar{\alpha}_{s}^{2}\left(\mu_{R}\right) \chi^{(1)}(\nu)\right]} \\
& \times F_{i}(\nu) F_{k}(-\nu)\left[1+\bar{\alpha}_{s}\left(\mu_{R}\right)\left(\frac{F_{i}^{(1)}(\nu)}{F_{i}(\nu)}+\frac{F_{k}^{(1)}(-\nu)}{F_{k}(-\nu)}\right)\right]
\end{aligned}
$$




\begin{tabular}{|llcclcc|}
\hline$Y$ & $\sigma_{\text {tot }}^{(\exp , 1)}[\mathrm{nb}]$ & $\mu_{R} / Q$ & $Y_{0}$ & $\sigma_{\text {tot }}^{(\exp , 2)}[\mathrm{nb}]$ & $\mu_{R} / Q$ & $Y_{0}$ \\
\hline 2 & 7.36281 & 18 & 1 & 7.57706 & 8 & 1 \\
3.5 & 3.23512 & 18 & 3 & 3.25243 & 8 & 1 \\
4.5 & 1.98923 & 18 & 4 & 1.9419 & 8 & 1 \\
6 & 1.20222 & 18 & 5 & 1.09588 & 8 & 1 \\
\hline
\end{tabular}

Table 2. Values of $\sigma_{\text {tot }}^{(\exp , 1,2)}$ for several values of $Y$ at $Q^{2}=17 \mathrm{GeV}^{2}$; the columns 3-4 and 6-7 give the optimal values of the renormalization and energy scales.

and

$$
\begin{gathered}
\sigma_{i k}^{(\exp , 2)=} \frac{1}{(2 \pi)^{2} Q^{2}} \int_{-\infty}^{+\infty} d \nu e^{\left(Y-Y_{0}\right)\left[\bar{\alpha}_{s}\left(\mu_{R}\right)\left(1+\frac{\bar{\alpha}_{s}\left(\mu_{R}\right) \beta_{0}}{4 N_{c}} \ln \frac{\mu_{R}^{2}}{Q^{2}}\right) \chi(\nu)+\bar{\alpha}_{s}^{2}\left(\mu_{R}\right) \chi^{(1)}(\nu)\right]} \\
\times F_{i}(\nu) F_{k}(-\nu)\left[1+\bar{\alpha}_{s}\left(\mu_{R}\right)\left(\frac{F_{i}^{(1)}(\nu)}{F_{i}(\nu)}+\frac{F_{k}^{(1)}(-\nu)}{F_{k}(-\nu)}\right)\right. \\
\left.+\bar{\alpha}_{s}^{2}\left(\mu_{R}\right)\left(\frac{F_{i}^{(1)}(\nu)}{F_{i}(\nu)} \frac{F_{k}^{(1)}(-\nu)}{F_{k}(-\nu)}\right)\right]
\end{gathered}
$$

In these equations we denote for shortness $F_{i, k}^{(1)}(\nu) \equiv F_{i, k}^{(1)}\left(\nu, s_{0}, \mu_{R}\right)$.

We used these two exponentiated representations together with the PMS method to fix the values of the energy scales and obtained the results given in table 2 . We can see that the variant (2) of the exponentiated cross section gets lower values for the optimal energy scales, thus implying that the inclusion of the subleading term with the product of the NLO impact factors catches a relevant part of the unknown next-to-NLA corrections. However, as shown in figure 5, the absolute value of the cross section remains low and undershoots LEP2 data substantially in the same fashion as the two previous representations.

\subsection{Exponential representation with BLM optimization}

Here we consider the first variant of the exponentiated cross section discussed in the previous subsection, combined with two different implementations (variants $(a)$ and $(b)$ ) of the BLM method (for a justification of the formulas below, we refer to [73]):

$$
\sigma_{\text {tot }}^{(\mathrm{BLM}, \mathrm{a})}(s, Q)=\sigma_{T T}^{(\mathrm{BLM}, \mathrm{a})}+\sigma_{L L}^{(\mathrm{BLM}, \mathrm{a})}+\sigma_{T L}^{(\mathrm{BLM}, \mathrm{a})}+\sigma_{L T}^{(\mathrm{BLM}, \mathrm{a})}+\sigma_{\mathrm{LO} \text { box }},
$$

where

$$
\begin{aligned}
\sigma_{i k}^{(\mathrm{BLM}, \mathrm{a})}= & \frac{1}{(2 \pi)^{2} Q^{2}} \int_{-\infty}^{+\infty} d \nu e^{\left(Y-Y_{0}\right)\left[\bar{\alpha}_{s}\left(\mu_{R, a}^{\mathrm{BLM}}\right) \chi(\nu)+\left(\bar{\alpha}_{s}\left(\mu_{R, a}^{\mathrm{BLM}}\right)\right)^{2}\left(-\frac{T^{\beta}}{N_{c}} \chi(\nu)+\bar{\chi}(\nu)-\frac{\beta_{0}}{8 N_{c}} \chi^{2}(\nu)\right)\right]} \\
& \times F_{i}(\nu) F_{k}(-\nu)\left[1+\bar{\alpha}_{s}\left(\mu_{R, a}^{\mathrm{BLM}}\right)\left(\frac{\tilde{F}_{i}^{(1)}(\nu)}{F_{i}(\nu)}+\frac{\tilde{F}_{k}^{(1)}(-\nu)}{F_{k}(-\nu)}-2 \frac{T^{\beta}}{N_{c}}\right)\right]
\end{aligned}
$$

with

$$
\left(\mu_{R, a}^{\mathrm{BLM}}\right)^{2}=Q^{2} \exp \left[2\left(1+\frac{2}{3} I\right)-\frac{5}{3}\right]
$$




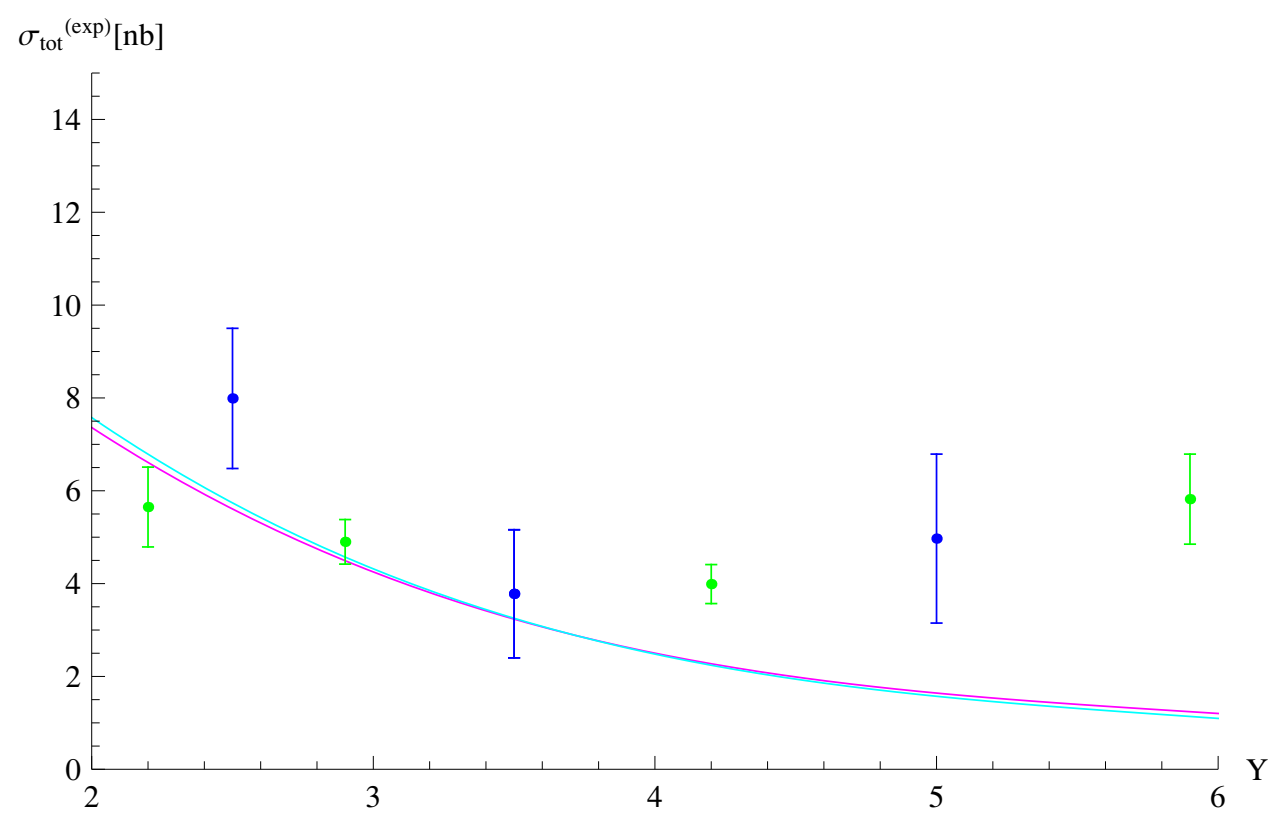

Figure 5. $\sigma_{\text {tot }}^{(\exp , 1)}$ (magenta line) and $\sigma_{\text {tot }}^{(\exp , 2)}$ (cyan line) versus $Y$ at $Q^{2}=17 \mathrm{GeV}^{2}\left(n_{f}=4\right)$, together with the experimental data from OPAL (blue points, $Q^{2}=18 \mathrm{GeV}^{2}$ ) and L3 (green points, $\left.Q^{2}=16 \mathrm{GeV}^{2}\right)$.

and

$$
\sigma_{\text {tot }}^{(\mathrm{BLM}, \mathrm{b})}(s, Q)=\sigma_{T T}^{(\mathrm{BLM}, \mathrm{b})}+\sigma_{L L}^{(\mathrm{BLM}, \mathrm{b})}+\sigma_{T L}^{(\mathrm{BLM}, \mathrm{b})}+\sigma_{L T}^{(\mathrm{BLM}, \mathrm{b})}+\sigma_{\mathrm{LO} \text { box }},
$$

where

$$
\begin{gathered}
\sigma_{i k}^{(\mathrm{BLM}, \mathrm{b})}=\frac{1}{(2 \pi)^{2} Q^{2}} \int_{-\infty}^{+\infty} d \nu e^{\left(Y-Y_{0}\right)\left[\bar{\alpha}_{s}\left(\mu_{R, b}^{\mathrm{BLM}}\right) \chi(\nu)+\left(\bar{\alpha}_{s}\left(\mu_{R, b}^{\mathrm{BLM}}\right)\right)^{2}\left(-\frac{T^{\beta}}{N_{c}} \chi(\nu)+\bar{\chi}(\nu)\right)\right]} \\
\times F_{i}(\nu) F_{k}(-\nu)\left[1+\bar{\alpha}_{s}\left(\mu_{R, b}^{\mathrm{BLM}}\right)\left(\frac{\tilde{F}_{i}^{(1)}(\nu)}{F_{i}(\nu)}+\frac{\tilde{F}_{k}^{(1)}(-\nu)}{F_{k}(-\nu)}\right)\right. \\
\left.+\bar{\alpha}_{s}\left(\mu_{R, b}^{\mathrm{BLM}}\right)\left(-\frac{2 T^{\beta}}{N_{c}}+\frac{\beta_{0}}{4 N_{c}} \chi(\nu)\right)\right]
\end{gathered}
$$

with

$$
\left(\mu_{R, b}^{\mathrm{BLM}}\right)^{2}=Q^{2} \exp \left[2\left(1+\frac{2}{3} I\right)-\frac{5}{3}+\frac{1}{2} \chi(\nu)\right]
$$

In eqs. (3.13) and (3.16) the LO impact factors have to be evaluated with $\alpha_{s}=\alpha_{s}\left(\mu_{R, a}^{\mathrm{BLM}}\right)$ and $\alpha_{s}=\alpha_{s}\left(\mu_{R, b}^{\mathrm{BLM}}\right)$ respectively. In both cases, we have

$$
T^{\beta}=-\frac{\beta_{0}}{2}\left[1+\frac{2}{3} I\right], \quad I \simeq 2.3439 .
$$

Besides, in eqs. (3.13) and (3.16) we denote

$$
\frac{\tilde{F}_{i}^{(1)}(\nu)}{F_{i}(\nu)} \equiv \frac{F_{i}^{(1)}\left(\nu, s_{0}, \mu_{R}\right)}{F_{i}(\nu)}-\frac{\beta_{0}}{4 N_{c}}\left(\ln \frac{\mu_{R}^{2}}{Q^{2}}+\frac{5}{3}\right) .
$$




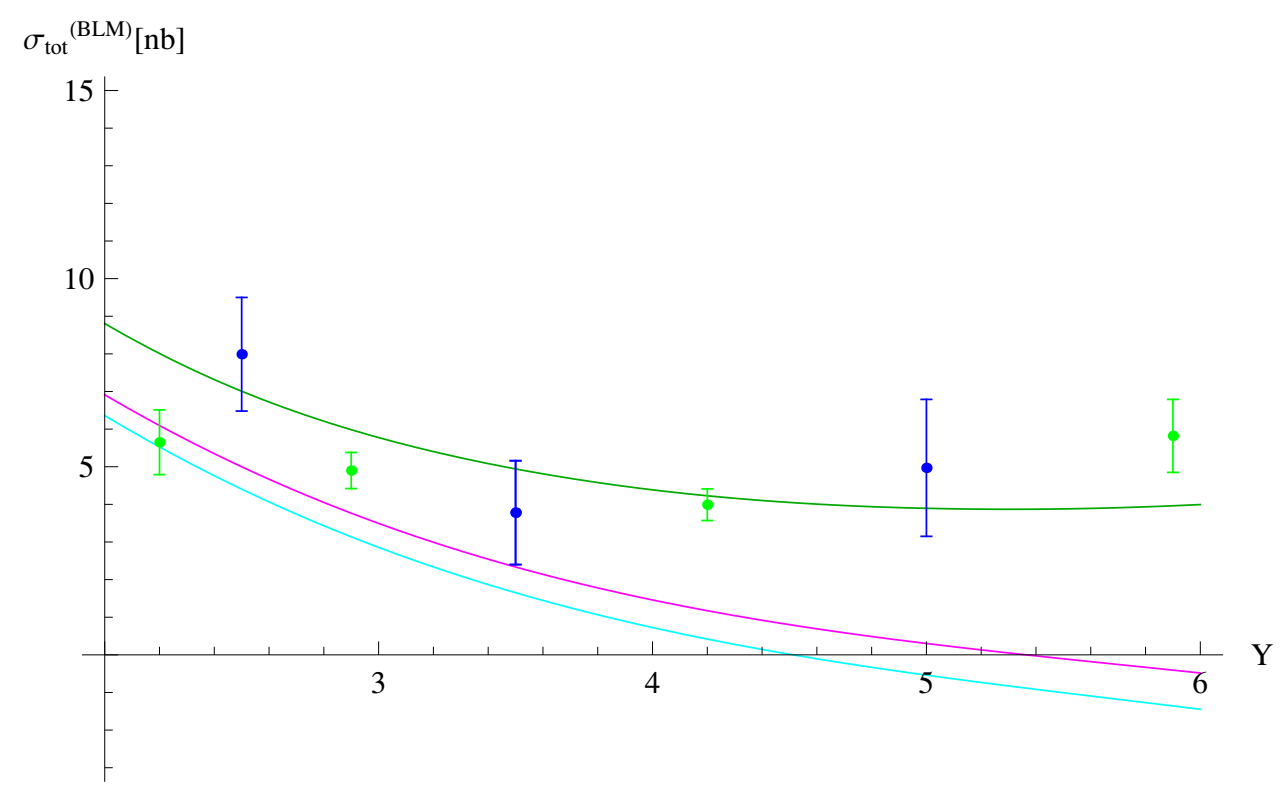

Figure 6. $\sigma_{\text {tot }}^{(\mathrm{BLM}, \text { a) }}$ (cyan line) and $\sigma_{\text {tot }}^{\text {(BLM, b) }}$ (magenta line) versus $Y$ at $Q^{2}=17 \mathrm{GeV}^{2}\left(n_{f}=4\right)$, together with the experimental data from OPAL (blue points, $Q^{2}=18 \mathrm{GeV}^{2}$ ) and L3 (green points, $Q^{2}=16 \mathrm{GeV}^{2}$ ); the green line represents the result of ref. [47] (see figure 3 there).

The results in this approach, calculated at $s_{0}=Q^{2}$, are shown in figure 6 , where we can see that the cross section is very low and starts even to be negative at larger values of $Y$. In the same figure, we show also the result obtained in ref. [47] by a similar approach, but in the approximation where photon impact factors were taken at the LO.

\section{Discussion}

In this paper we have studied the $\gamma^{*} \gamma^{*}$ total cross section in the NLA BFKL approach. First we have extracted the NLO corrections to the photon impact factor from two recent papers $[49,50]$, then we have used them to build several representations of the total cross section, equivalent within the NLA, but taking into account in a different way pieces of the (unknown) subleading contributions. We have combined these different representations with two among the most common methods for the optimization of a perturbative series, namely PMS and BLM, and compared their behavior with the energy with the only available experimental data, those from the LEP2 collider. We have considered also the numerical implementation of formulas describing the BFKL contribution to $\gamma^{*} \gamma^{*}$ total cross section, derived originally by Chirilli and Kovchegov [50].

We have found that, in general, the effect of the BFKL resummation is small and changes only by little the determination coming from the LO quark box diagrams. This means that, in the considered range of energies, the NLO corrections to the photon impact factor compensate almost exactly the LO ones. Indeed, previous estimates of the cross section [45-48] using LO impact factors together with the NLA BFKL Green's function showed a better agreement with LEP2 data. 


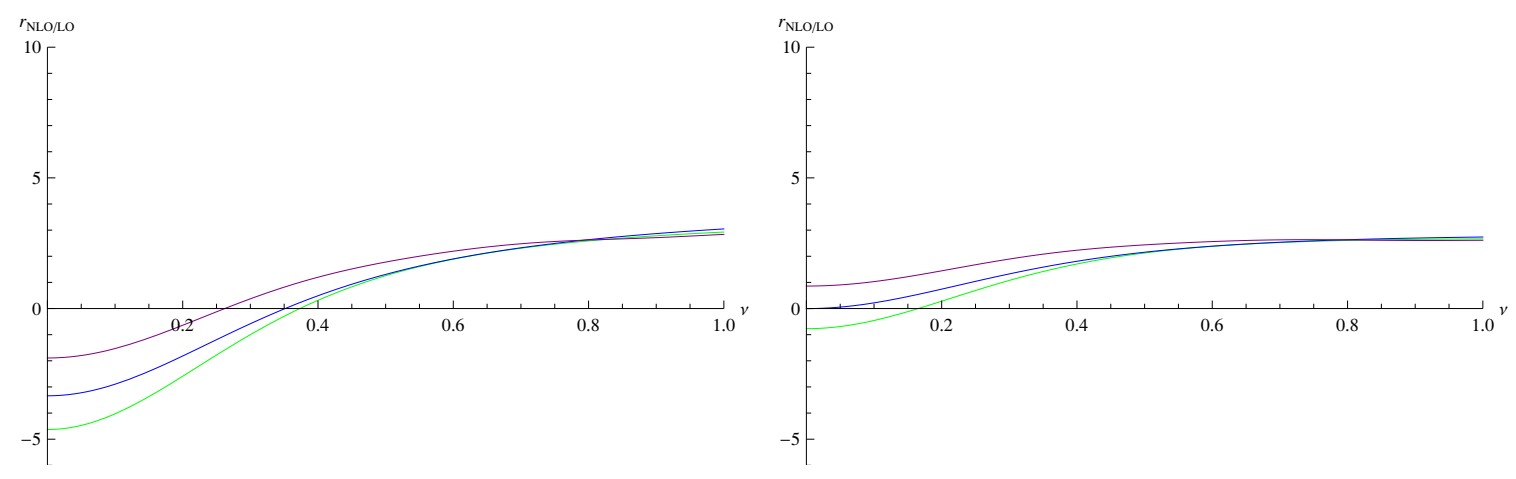

Figure 7. Behavior of $r_{\mathrm{NLO} / \mathrm{LO}}^{T}\left(\nu, s_{0}, \mu_{R}\right)$ (green), $r_{\mathrm{NLO} / \mathrm{LO}}^{L}\left(\nu, s_{0}, \mu_{R}\right)$ (blue) and $r_{\mathrm{NLO} / \mathrm{LO}}^{\text {(mesons) }}\left(\nu, s_{0}, \mu_{R}\right)$ (violet) for the following cases: $Q^{2}=\mu_{R}^{2}=17 \mathrm{GeV}^{2}, Y_{0}=0$ on the left and $Q^{2}=17 \mathrm{GeV}^{2}$, $\mu_{R}^{2}=(10 Q)^{2}, Y_{0}=2.2$ on the right.

In other words, the account of the Balitsky and Chirilli expression for NLO photon impact factor reduces the BFKL contribution to the cross section to very small values making it impossible to describe LEP2 data as a sum of BFKL and LO QED quark box contributions. Note that, the LO QED quark box itself receives, at higher QCD orders, large corrections enhanced by double logs. Their resummation is important and leads to a considerable enhancement of the quark box contribution - see ref. $[4,5]$ for details, but still these effects are not large enough for a good description of LEP2 data at $Y=3.5 \div 6$ without a sizable BFKL contribution.

There could be many reasons for this problem at $Y=3.5 \div 6$. The first, obvious one, is that even at such high energies the BFKL contribution could be still not the dominant one in comparison with terms which are suppressed by powers of the energy $\sim 1 / s$, and are not included in the present consideration. In particular, terms, subleading in energy, coming from diagrams with gluon exchange in the $t$-channel, see figure 2 , can be important. We could also argue that the presumably large effects in the next-to-NLA are not reduced under enough satisfactory control by the representations of the cross section and by the optimization methods we have considered in this work. In this respect, it would be interesting to test also approaches based on collinear improvement [74-84]. However, the consideration of these issues goes beyond the scope of present paper.

Definitely, the problems with our description of LEP2 data in the present context originate from the large negative value of NLO contributions to the photon impact factor. For this reason, we will discuss several issues related with this quantity. First, we want to illustrate our statement that the NLO corrections to the photon impact factor turned to be very large. For this purpose we plot in figure 7 the factors which, in the case of transverse and longitudinal photon polarizations, control the normalization of the cross section in the case of the exponential representation (3.10),

$$
r_{\mathrm{NLO} / \mathrm{LO}}^{(T, L)}\left(\nu, s_{0}, \mu_{R}\right) \equiv 1+\bar{\alpha}_{s}\left(\mu_{R}\right)\left(\frac{F_{T, L}^{(1)}\left(\nu, s_{0}, \mu_{R}\right)}{F_{T, L}(\nu)}+\frac{F_{T, L}^{(1)}\left(-\nu, s_{0}, \mu_{R}\right)}{F_{T, L}(-\nu)}\right) .
$$

For the sake of comparison, we present in figure 7 also the similar quantity $r_{\mathrm{NLO} / \mathrm{LO}}^{\text {(mesons }}$ which 
appeared in the description of the process $\gamma^{*} \gamma^{*}$ to two light vector mesons, see refs. [57-59],

$$
r_{\mathrm{NLO} / \mathrm{LO}}^{(\mathrm{mesons})}\left(\nu, s_{0}, \mu_{R}\right) \equiv 1+\bar{\alpha}_{s}\left(\mu_{R}\right)\left(\frac{c_{1}^{(1)}\left(\nu, s_{0}, \mu_{R}\right)}{c_{1}(\nu)}+\frac{c_{2}^{(1)}\left(-\nu, s_{0}, \mu_{R}\right)}{c_{2}(-\nu)}\right) .
$$

The $\nu$ dependence of these quantities is shown on the left panel of figure 7 in the case of natural scale choice, $s_{0}=\mu_{R}^{2}=Q^{2}$, whereas on the right panel we show the same quantities calculated for $\mu_{R}^{2}=(10 Q)^{2}, Y_{0}=2.2$, the values of scales which were obtained in refs. [5759] during PMS optimization procedure applied to $\gamma^{*} \gamma^{*} \rightarrow V V$ process. In the region of large- $\nu$ the results are similar in all the three cases, whereas in the low- $\nu$ region they differ substantially. For natural scales (left panel) and $\nu \leq 0.25$ all the three quantities are negative; note that it is the region of $\nu$ that dominates the $\nu$-integral appearing in the cross section. We see that in this $\nu$-region the NLO corrections to the impact factors are negative and turn to be much larger for $\gamma^{*} \rightarrow \gamma^{*}$ (especially in the case of transverse polarization) in comparison to the case of $\gamma^{*} \rightarrow V$ impact factor. Such a difference remains to be understood.

The impact factors in BFKL approach depend on the scales $s_{0}$ and $\mu_{R}$, see eqs. (2.12) and (2.13). Comparing the left and right panels of figure 7 , one can see that this effect is important. In particular, $\gamma^{*} \rightarrow V$ and $\gamma_{L}^{*} \rightarrow \gamma_{L}^{*}$ impact factors become positive in the whole $\nu$ range when one goes from the natural choice of scales to $\mu_{R}^{2}=(10 Q)^{2}, Y_{0}=2.2$. But it is not the case for the $\gamma_{T}^{*} \rightarrow \gamma_{T}^{*}$ impact factor, which remains negative-valued for a substantial range of small $\nu$. Note that the transverse polarization gives the most important contributions $\left(\sigma_{T T}\right)$ to the effective $\gamma^{*} \gamma^{*}$ cross section which we consider in this paper. This observation explains, on the qualitative level, the very high values of optimal scales in tables 1 and 2, which we found with PMS method for the $\gamma^{*} \gamma^{*}$ total cross section.

The other issue we want to mention here is the color structure of the NLO parts of the photon impact factors. We observe that the NLO impact factors as extracted from [50] have very simple subleading $\sim 1 / N_{c}^{2}$ contributions, which appear only in the trivial third terms of eqs. (2.12) and (2.13). This is in sharp contrast with what happens in the case of the NLO virtual photon to light vector meson impact factor [56] and of the NLO forward jet impact factor [60-63]. It would be interesting to understand the reason for the practically complete cancellation of the subleading $1 / N_{c}^{2}$ terms which takes place here.

Finally, we want to compare the results for the photon impact factor which we used in this paper (derived from the results in $[49,50]$ ) with the ones obtained in the conventional BFKL approach by Bartels and collaborators [38-44]. Unfortunately, some information (in numerical form) about the final result for the impact factor is available only for the case of transverse polarization - see ref. [85] in the "Diffraction 2006" workshop proceedings. To make such a comparison we need to transfer the photon impact factor from the $\nu$ - to the transverse momentum representation:

$$
\Phi_{T}\left(x, s_{0}, \mu_{R}\right)=\int_{-\infty}^{\infty} d \nu \frac{(x)^{-i \nu+\frac{1}{2}}}{\pi \sqrt{2}}\left[F_{T}(\nu)+\bar{\alpha}_{s}\left(\mu_{R}\right) F_{T}^{(1)}\left(\nu, s_{0}, \mu_{R}\right)\right],
$$

where the variable $x$ is defined as a dimensionless ratio of the Reggeon transverse momentum $\vec{q}$ and the photon virtuality squared: $x \equiv \vec{q}^{2} / Q^{2}$. The plot of $\Phi_{T}\left(x, s_{0}, \mu_{R}\right)$ as 


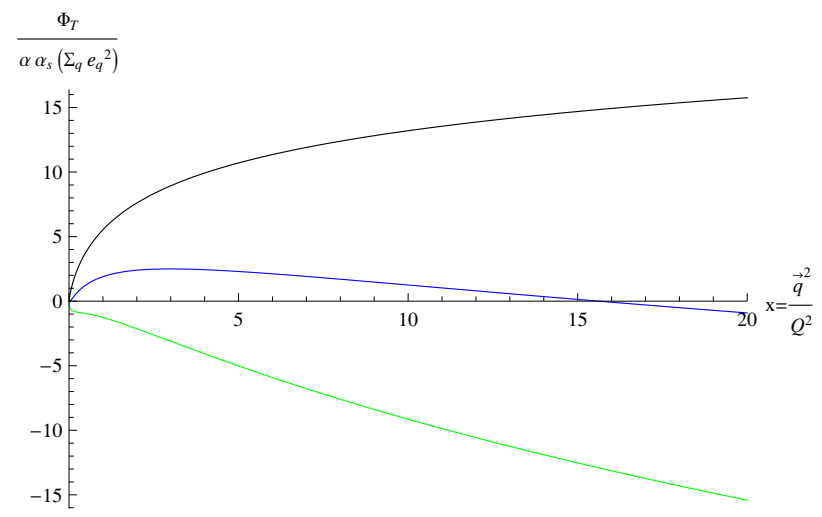

Figure 8. Behavior of the photon impact factor (the transverse polarization) with the Reggeon transverse momentum $\vec{q}$, through the variable $x \equiv \vec{q}^{2} / Q^{2}$. The black curve represents the LO impact factor, the green curve the sum of LO and NLO parts derived from eq. (2.12) and the blue curve the same as the green curve, but with the NLO part reduced by the factor 1.87.

a function of $x$ is presented in figure 6 of ref. [85]. Here we perform the $\nu$ integration in (4.3) using eq. (2.12), the Balitsky-Chirilli result for the transverse photon impact factor transformed to the conventional BFKL scheme. In our figure 8 we present results for $\Phi_{T}\left(x, s_{0}, \mu_{R}\right) /\left(\alpha \alpha_{s}\left(\sum_{q} e_{q}^{2}\right)\right)$, where we used the following settings in order to compare with [85]: $s_{0}=10 \mathrm{GeV}^{2}, Q^{2}=\mu_{R}^{2}=15 \mathrm{GeV}^{2}$; moreover, we take $n_{f}=1$ and $\alpha_{s}=0.177206 .^{2}$ In figure 8 we show the behavior of the photon impact factor with the Reggeon transverse momentum $\vec{q}$, through the variable $x$. In figure 8 the black curve represents the LO impact factor, the green curve gives LO plus NLO parts derived from eq. (2.12), and the blue curve LO plus NLO parts derived from eq. (2.12), when NLO contribution is reduced by the factor 1.87 . We see that the NLO corrections are rather large and it is clear that the $x$-shape of $\Phi\left(x, s_{0}, \mu_{R}\right)$ is rather sensitive to their value. Comparing the shape of the $x$-dependence in figure 6 of [85] with the NLO curves in figure 8 , we should conclude that the results of Balitsky and Chirilli are not in agreement with those presented in [85]. Interestingly, a qualitative agreement for the $x$-shape of $\Phi\left(x, s_{0}, \mu_{R}\right)$ could be obtained only reducing the NLO result given in eq. $(2.12)$ by the factor $\sim 1.87$.

To summarize this discussion we would like to stress that it would be very important if the authors of [85] could finally publish their results for the photon impact factor, since it would be an independent test of the results obtained by Balitsky and Chirilli using a completely different approach.

\section{Acknowledgments}

The authors are grateful to G. Chirilli and Yu. Kovchegov for valuable discussions. D.I. thanks the Dipartimento di Fisica dell'Università della Calabria and the Istituto Nazionale di Fisica Nucleare (INFN), Gruppo collegato di Cosenza, for warm hospitality and financial

\footnotetext{
${ }^{2}$ We are grateful to Grigorios Chachamis who provided us with the information about the numeric values of $n_{f}$ and $\alpha_{s}$ which were used to produce figure 6 of [85].
} 
support. The work of D.I. was also supported in part by the grant RFBR-13-02-00695-a. The work of B.M. was supported by the European Commission, European Social Fund and Calabria Region, that disclaim any liability for the use that can be done of the information provided in this paper.

B.M. thanks the Sobolev Institute of Mathematics of Novosibirsk for warm hospitality during the preparation of this work.

Open Access. This article is distributed under the terms of the Creative Commons Attribution License (CC-BY 4.0), which permits any use, distribution and reproduction in any medium, provided the original author(s) and source are credited.

\section{References}

[1] V.M. Budnev, I.F. Ginzburg, G.V. Meledin and V.G. Serbo, The Two photon particle production mechanism. Physical problems. Applications. Equivalent photon approximation, Phys. Rept. 15 (1975) 181 [INSPIRE].

[2] I. Schienbein, Two photon processes and photon structure, Annals Phys. 301 (2002) 128 [hep-ph/0205301] [INSPIRE].

[3] M. Cacciari, V. Del Duca, S. Frixione and Z. Trócsányi, QCD radiative corrections to $\gamma^{*} \gamma^{*} \rightarrow$ hadrons, JHEP 02 (2001) 029 [hep-ph/0011368] [INSPIRE].

[4] J. Bartels and M. Lublinsky, Quark anti-quark exchange in $\gamma^{*} \gamma^{*}$ scattering, JHEP 09 (2003) 076 [hep-ph/0308181] [INSPIRE].

[5] J. Bartels and M. Lublinsky, $\gamma^{*} \gamma^{*}$ scattering via secondary Reggeon exchange in QCD, Mod. Phys. Lett. A 19 (2004) 19691982 [hep-ph/0406273] [INSPIRE].

[6] V.S. Fadin, E.A. Kuraev and L.N. Lipatov, On the Pomeranchuk Singularity in Asymptotically Free Theories, Phys. Lett. B 60 (1975) 50 [inSPIRE].

[7] E.A. Kuraev, L.N. Lipatov and V.S. Fadin, Multi-Reggeon Processes in the Yang-Mills Theory, Zh. Eksp. Teor. Fiz. 71 (1976) 840 [Sov. Phys. JETP 44 (1976) 443] [inSPIRE].

[8] E.A. Kuraev, L.N. Lipatov and V.S. Fadin, The Pomeranchuk Singularity in Nonabelian Gauge Theories, Zh. Eksp. Teor. Fiz. 72 (1977) 377 [Sov. Phys. JETP 45 (1977) 199] [INSPIRE].

[9] I.I. Balitsky and L.N. Lipatov, The Pomeranchuk Singularity in Quantum Chromodynamics, Sov. J. Nucl. Phys. 28 (1978) 822 [inSPIRE].

[10] F. Hautmann, The Total cross-section for off-shell photons at high-energies, OITS-613-96, C96-07-25.

[11] J. Bartels, A. De Roeck and H. Lotter, The $\gamma^{*} \gamma^{*}$ total cross-section and the BFKL Pomeron at $e^{+} e^{-}$colliders, Phys. Lett. B 389 (1996) 742 [hep-ph/9608401] [INSPIRE].

[12] A. Bialas, W. Czyz and W. Florkowski, Total $\gamma^{*} \gamma^{*}$ cross-section and the BFKL Pomeron, Eur. Phys. J. C 2 (1998) 683 [hep-ph/9705470] [INSPIRE].

[13] S.J. Brodsky, F. Hautmann and D.E. Soper, Virtual photon scattering at high-energies as a probe of the short distance Pomeron, Phys. Rev. D 56 (1997) 6957 [hep-ph/9706427] [INSPIRE]. 
[14] S.J. Brodsky, F. Hautmann and D.E. Soper, Probing the QCD Pomeron in $e^{+} e^{-}$collisions, Phys. Rev. Lett. 78 (1997) 803 [Erratum ibid. 79 (1997) 3544] [hep-ph/9610260] [INSPIRE].

[15] J. Kwiecinski and L. Motyka, Probing the QCD Pomeron in doubly tagged $e^{+} e^{-}$collisions, Phys. Lett. B 462 (1999) 203 [hep-ph/9905567] [INSPIRE].

[16] J. Kwiecinski and L. Motyka, Theoretical description of the total gamma* gamma* cross-section and its confrontation with the LEP data on doubly tagged $e^{+} e^{-}$events, Eur. Phys. J. C 18 (2000) 343 [hep-ph/0010029] [inSPIRE].

[17] M. Boonekamp, A. De Roeck, C. Royon and S. Wallon, $\gamma^{*} \gamma^{*}$ total cross-section in the dipole picture of BFKL dynamics, Nucl. Phys. B 555 (1999) 540 [hep-ph/9812523] [INSPIRE].

[18] J. Bartels, C. Ewerz and R. Staritzbichler, Effect of the charm quark mass on the BFKL $\gamma^{*} \gamma^{*}$ total cross-section at LEP, Phys. Lett. B 492 (2000) 56 [hep-ph/0004029] [INSPIRE].

[19] N.N. Nikolaev, J. Speth and V.R. Zoller, Predictions for high-energy real and virtual photon-photon scattering from color dipole BFKL-Regge factorization, Eur. Phys. J. C 22 (2002) 637 [hep-ph/0001120] [INSPIRE].

[20] N.N. Nikolaev, J. Speth and V.R. Zoller, Color dipole BFKL-Regge factorization and high-energy photon photon scattering, J. Exp. Theor. Phys. 93 (2001) 957 [INSPIRE].

[21] V.S. Fadin and L.N. Lipatov, BFKL Pomeron in the next-to-leading approximation, Phys. Lett. B 429 (1998) 127 [hep-ph/9802290] [INSPIRE].

[22] M. Ciafaloni and G. Camici, Energy scale(s) and next-to-leading BFKL equation, Phys. Lett. B 430 (1998) 349 [hep-ph/9803389] [INSPIRE].

[23] L.N. Lipatov and V.S. Fadin, High-Energy Production of Gluons in a QuasimultiRegge Kinematics, JETP Lett. 49 (1989) 352 [INSPIRE].

[24] V.S. Fadin and R. Fiore, Quark contribution to the gluon-gluon - reggeon vertex in QCD, Phys. Lett. B 294 (1992) 286 [INSPIRE].

[25] V.S. Fadin and L.N. Lipatov, Radiative corrections to QCD scattering amplitudes in a multi - Regge kinematics, Nucl. Phys. B 406 (1993) 259 [InSPIRE].

[26] V.S. Fadin, R. Fiore and A. Quartarolo, Quark contribution to the reggeon - reggeon - gluon vertex in QCD, Phys. Rev. D 50 (1994) 5893 [hep-th/9405127] [INSPIRE].

[27] V.S. Fadin, M.I. Kotsky and R. Fiore, Gluon Reggeization in QCD in the next-to-leading order, Phys. Lett. B 359 (1995) 181 [INSPIRE].

[28] V.S. Fadin, R. Fiore and M.I. Kotsky, Gluon Regge trajectory in the two loop approximation, Phys. Lett. B 387 (1996) 593 [hep-ph/9605357] [INSPIRE].

[29] V.S. Fadin, R. Fiore and M.I. Kotsky, Gribov's theorem on soft emission and the reggeon-reggeon - gluon vertex at small transverse momentum, Phys. Lett. B 389 (1996) 737 [hep-ph/9608229] [INSPIRE].

[30] V.S. Fadin, R. Fiore and A. Quartarolo, Reggeization of quark quark scattering amplitude in QCD, Phys. Rev. D 53 (1996) 2729 [hep-ph/9506432] [INSPIRE].

[31] V.S. Fadin and L.N. Lipatov, Next-to-leading corrections to the BFKL equation from the gluon and quark production, Nucl. Phys. B 477 (1996) 767 [hep-ph/9602287] [INSPIRE].

[32] V.S. Fadin, M.I. Kotsky and L.N. Lipatov, One-loop correction to the BFKL kernel from two gluon production, Phys. Lett. B $4 \mathbf{1 5}$ (1997) 97 [INSPIRE]. 
[33] V.S. Fadin, R. Fiore, A. Flachi and M.I. Kotsky, Quark - anti-quark contribution to the BFKL kernel, Phys. Lett. B 422 (1998) 287 [hep-ph/9711427] [INSPIRE].

[34] S. Catani, M. Ciafaloni and F. Hautmann, Gluon contributions to small x heavy flavor production, Phys. Lett. B 242 (1990) 97 [INSPIRE].

[35] G. Camici and M. Ciafaloni, Non-Abelian q $q \bar{q}$ contributions to small $x$ anomalous dimensions, Phys. Lett. B 386 (1996) 341 [hep-ph/9606427] [INSPIRE].

[36] G. Camici and M. Ciafaloni, $k$ factorization and small $x$ anomalous dimensions, Nucl. Phys. B 496 (1997) 305 [Erratum ibid. B 607 (2001) 431] [hep-ph/9701303] [INSPIRE].

[37] V.S. Fadin, BFKL news, hep-ph/9807528 [INSPIRE].

[38] J. Bartels, S. Gieseke and C.F. Qiao, The $\left(\gamma^{*} \rightarrow q \bar{q}\right)$ Reggeon vertex in next-to-leading order QCD, Phys. Rev. D 63 (2001) 056014 [Erratum ibid. D 65 (2002) 079902] [hep-ph/0009102] [INSPIRE].

[39] J. Bartels, S. Gieseke and A. Kyrieleis, The Process $\gamma_{L}^{*}+q \rightarrow(q \bar{q} g)+q:$ Real corrections to the virtual photon impact factor, Phys. Rev. D 65 (2002) 014006 [hep-ph/0107152] [INSPIRE].

[40] J. Bartels, D. Colferai, S. Gieseke and A. Kyrieleis, NLO corrections to the photon impact factor: Combining real and virtual corrections, Phys. Rev. D 66 (2002) 094017 [hep-ph/0208130] [INSPIRE].

[41] J. Bartels, The photon impact factor in next-to-leading order, Nucl. Phys. Proc. Suppl. 116 (2003) 126 [INSPIRE].

[42] J. Bartels and A. Kyrieleis, NLO corrections to the $\gamma^{*}$ impact factor: First numerical results for the real corrections to $\gamma_{L}^{*}$, Phys. Rev. D 70 (2004) 114003 [hep-ph/0407051] [INSPIRE].

[43] V.S. Fadin, D.Yu. Ivanov and M.I. Kotsky, Photon Reggeon interaction vertices in the NLA, Phys. Atom. Nucl. 65 (2002) 1513 [hep-ph/0106099] [INSPIRE].

[44] V.S. Fadin, D.Yu. Ivanov and M.I. Kotsky, On the calculation of the NLO virtual photon impact factor, Nucl. Phys. B 658 (2003) 156 [hep-ph/0210406] [INSPIRE].

[45] S.J. Brodsky, V.S. Fadin, V.T. Kim, L.N. Lipatov and G.B. Pivovarov, High-energy QCD asymptotics of photon-photon collisions, JETP Lett. 76 (2002) 249 [hep-ph/0207297] [INSPIRE].

[46] S.J. Brodsky, V.S. Fadin, V.T. Kim, L.N. Lipatov and G.B. Pivovarov, The QCD Pomeron with optimal renormalization, JETP Lett. 70 (1999) 155 [hep-ph/9901229] [INSPIRE].

[47] F. Caporale, D.Yu. Ivanov and A. Papa, BFKL resummation effects in the $\gamma^{*} \gamma^{*}$ total hadronic cross section, Eur. Phys. J. C 58 (2008) 1 [arXiv:0807.3231] [INSPIRE].

[48] X.-C. Zheng, X.-G. Wu, S.-Q. Wang, J.-M. Shen and Q.-L. Zhang, Reanalysis of the BFKL Pomeron at the next-to-leading logarithmic accuracy, JHEP 10 (2013) 117 [arXiv: 1308.2381] [INSPIRE].

[49] I. Balitsky and G.A. Chirilli, Photon impact factor and $k_{T}$-factorization for DIS in the next-to-leading order, Phys. Rev. D 87 (2013) 014013 [arXiv: 1207.3844] [INSPIRE].

[50] G.A. Chirilli and Y.V. Kovchegov, $\gamma^{*} \gamma^{*}$ Cross Section at NLO and Properties of the BFKL Evolution at Higher Orders, JHEP 05 (2014) 099 [arXiv:1403.3384] [INSPIRE]. 
[51] V.S. Fadin, R. Fiore and A. Papa, The Dipole form of the quark part of the BFKL kernel, Phys. Lett. B 647 (2007) 179 [hep-ph/0701075] [INSPIRE].

[52] V.S. Fadin, R. Fiore and A. Papa, On the coordinate representation of NLO BFKL, Nucl. Phys. B 769 (2007) 108 [hep-ph/0612284] [INSPIRE].

[53] V.S. Fadin, R. Fiore, A.V. Grabovsky and A. Papa, The Dipole form of the gluon part of the BFKL kernel, Nucl. Phys. B 784 (2007) 49 [arXiv:0705.1885] [InSPIRE].

[54] I. Balitsky and G.A. Chirilli, High-energy amplitudes in $N=4$ SYM in the next-to-leading order, Phys. Lett. B 687 (2010) 204 [arXiv:0911.5192] [INSPIRE].

[55] G.A. Chirilli and Y.V. Kovchegov, Solution of the NLO BFKL Equation and a Strategy for Solving the All-Order BFKL Equation, JHEP 06 (2013) 055 [arXiv:1305.1924] [INSPIRE].

[56] D.Yu. Ivanov, M.I. Kotsky and A. Papa, The Impact factor for the virtual photon to light vector meson transition, Eur. Phys. J. C 38 (2004) 195 [hep-ph/0405297] [INSPIRE].

[57] D.Yu. Ivanov and A. Papa, Electroproduction of two light vector mesons in the next-to-leading approximation, Nucl. Phys. B 732 (2006) 183 [hep-ph/0508162] [INSPIRE].

[58] D.Yu. Ivanov and A. Papa, Electroproduction of two light vector mesons in next-to-leading BFKL: Study of systematic effects, Eur. Phys. J. C 49 (2007) 947 [hep-ph/0610042] [INSPIRE].

[59] F. Caporale, A. Papa and A. Sabio Vera, Collinear improvement of the BFKL kernel in the electroproduction of two light vector mesons, Eur. Phys. J. C 53 (2008) 525 [arXiv:0707.4100] [INSPIRE].

[60] J. Bartels, D. Colferai and G.P. Vacca, The NLO jet vertex for Mueller-Navelet and forward jets: The Quark part, Eur. Phys. J. C 24 (2002) 83 [hep-ph/0112283] [INSPIRE].

[61] J. Bartels, D. Colferai and G.P. Vacca, The NLO jet vertex for Mueller-Navelet and forward jets: The Gluon part, Eur. Phys. J. C 29 (2003) 235 [hep-ph/0206290] [InSPIRE].

[62] F. Caporale, D.Yu. Ivanov, B. Murdaca, A. Papa and A. Perri, The next-to-leading order jet vertex for Mueller-Navelet and forward jets revisited, JHEP 02 (2012) 101 [arXiv:1112.3752] [INSPIRE].

[63] D.Yu. Ivanov and A. Papa, The next-to-leading order forward jet vertex in the small-cone approximation, JHEP 05 (2012) 086 [arXiv:1202.1082] [INSPIRE].

[64] D. Colferai, F. Schwennsen, L. Szymanowski and S. Wallon, Mueller Navelet jets at LHC complete NLL BFKL calculation, JHEP 12 (2010) 026 [arXiv:1002.1365] [INSPIRE].

[65] B. Ducloué, L. Szymanowski and S. Wallon, Confronting Mueller-Navelet jets in NLL BFKL with LHC experiments at $7 \mathrm{TeV}$, JHEP 05 (2013) 096 [arXiv:1302.7012] [INSPIRE].

[66] F. Caporale, D.Yu. Ivanov, B. Murdaca and A. Papa, Mueller-Navelet small-cone jets at LHC in next-to-leading BFKL, Nucl. Phys. B 877 (2013) 73 [arXiv:1211.7225] [InSPIRE].

[67] B. Ducloué, L. Szymanowski and S. Wallon, Evidence for high-energy resummation effects in Mueller-Navelet jets at the LHC, Phys. Rev. Lett. 112 (2014) 082003 [arXiv:1309.3229] [INSPIRE].

[68] P.M. Stevenson, Resolution of the Renormalization Scheme Ambiguity in Perturbative QCD, Phys. Lett. B 100 (1981) 61 [INSPIRE].

[69] P.M. Stevenson, Optimized Perturbation Theory, Phys. Rev. D 23 (1981) 2916 [InSPIRE]. 
[70] S.J. Brodsky, G.P. Lepage and P.B. Mackenzie, On the Elimination of Scale Ambiguities in Perturbative Quantum Chromodynamics, Phys. Rev. D 28 (1983) 228 [InSPIRE].

[71] L3 collaboration, P. Achard et al., Double tag events in two photon collisions at LEP, Phys. Lett. B 531 (2002) 39 [hep-ex/0111012] [INSPIRE].

[72] OPAL collaboration, G. Abbiendi et al., Measurement of the hadronic cross-section for the scattering of two virtual photons at LEP, Eur. Phys. J. C 24 (2002) 17 [hep-ex/0110006] [INSPIRE].

[73] F. Caporale, D.Yu. Ivanov, B. Murdaca and A. Papa, On the BLM optimal renormalization scale setting for semihard processes, in preparation.

[74] G.P. Salam, A Resummation of large subleading corrections at small x, JHEP 07 (1998) 019 [hep-ph/9806482] [INSPIRE].

[75] M. Ciafaloni, D. Colferai, G.P. Salam and A.M. Stasto, The Gluon splitting function at moderately small x, Phys. Lett. B 587 (2004) 87 [hep-ph/0311325] [INSPIRE].

[76] M. Ciafaloni, D. Colferai, G.P. Salam and A.M. Stasto, Renormalization group improved small x Green's function, Phys. Rev. D 68 (2003) 114003 [hep-ph/0307188] [INSPIRE].

[77] M. Ciafaloni, D. Colferai, D. Colferai, G.P. Salam and A.M. Stasto, Extending QCD perturbation theory to higher energies, Phys. Lett. B 576 (2003) 143 [hep-ph/0305254] [INSPIRE].

[78] M. Ciafaloni, D. Colferai, G.P. Salam and A.M. Stasto, Tunneling transition to the Pomeron regime, Phys. Lett. B 541 (2002) 314 [hep-ph/0204287] [INSPIRE].

[79] M. Ciafaloni, D. Colferai, G.P. Salam and A.M. Stasto, Expanding running coupling effects in the hard Pomeron, Phys. Rev. D 66 (2002) 054014 [hep-ph/0204282] [INSPIRE].

[80] M. Ciafaloni, D. Colferai and G.P. Salam, On factorization at small x, JHEP 07 (2000) 054 [hep-ph/0007240] [INSPIRE].

[81] M. Ciafaloni, D. Colferai and G.P. Salam, A collinear model for small $x$ physics, JHEP 10 (1999) 017 [hep-ph/9907409] [INSPIRE].

[82] M. Ciafaloni, D. Colferai and G.P. Salam, Renormalization group improved small x equation, Phys. Rev. D 60 (1999) 114036 [hep-ph/9905566] [INSPIRE].

[83] M. Ciafaloni and D. Colferai, The BFKL equation at next-to-leading level and beyond, Phys. Lett. B 452 (1999) 372 [hep-ph/9812366] [INSPIRE].

[84] A. Sabio Vera, An 'All-poles' approximation to collinear resummations in the Regge limit of perturbative QCD, Nucl. Phys. B 722 (2005) 65 [hep-ph/0505128] [INSPIRE].

[85] G. Chachamis and J. Bartels, NLO Photon Impact Factor: Present Status and Outlook, PoS (DIFF2006) 026. 